QUANTUM PROBABILITY

BANACH CENTER PUBLICATIONS, VOLUME 73

INSTITUTE OF MATHEMATICS

POLISH ACADEMY OF SCIENCES

WARSZAWA 2006

\title{
A FREE ANALOGUE OF THE TRANSPORTATION COST INEQUALITY ON THE CIRCLE
}

\author{
FUMIO HIAI ${ }^{1,2}$ \\ Graduate School of Information Sciences, Tohoku University \\ Aoba-ku, Sendai 980-8579, Japan \\ E-mail: hiai@math.is.tohoku.ac.jp \\ DÉNES PETZ ${ }^{1,3}$ \\ Alfréd Rényi Institute of Mathematics, Hungarian Academy of Sciences \\ Reáltanoda u. 13-15, H-1053 Budapest, Hungary \\ E-mail:petz@math.bme.hu
}

Dedicated to Professor András Prékopa on the occasion of his 75th birthday

The relative entropy

$$
S(\mu, \nu):= \begin{cases}\int \log \frac{d \mu}{d \nu} d \mu & \text { if } \mu \ll \nu, \\ +\infty & \text { otherwise }\end{cases}
$$

and the Wasserstein distance are useful distances between measures. For probability measures $\mu$ and $\nu$ on the Euclidean space $\mathbb{R}^{n}$, the latter is defined as

$$
W(\mu, \nu):=\inf _{\pi \in \Pi(\mu, \nu)} \sqrt{\iint \frac{1}{2} d(x, y)^{2} d \pi(x, y)},
$$

where $d(x, y)=\|x-y\|_{2}$ and $\Pi(\mu, \nu)$ denotes the set of all probability measures on $\mathbb{R}^{n} \times \mathbb{R}^{n}$ with marginals $\mu$ and $\nu$, i.e., $\pi\left(\cdot \times \mathbb{R}^{n}\right)=\mu$ and $\pi\left(\mathbb{R}^{n} \times \cdot\right)=\nu$.

The transportation cost inequality (TCI) obtained by M. Talagrand [15] is

$$
W(\mu, \nu) \leq \sqrt{S(\mu, \nu)}
$$

2000 Mathematics Subject Classification: Primary 46L54; Secondary 60E15, 94A17, 15A52.

${ }^{1}$ Supported in part by Japan-Hungary JSPS-HAS Joint Project.

${ }^{2}$ Supported in part by Strategic Information and Communications R\&D Promotion Scheme of MPHPT.

${ }^{3}$ Supported in part by OTKA T032662.

The paper is in final form and no version of it will be published elsewhere. 
where $\nu$ is the standard Gaussian measure and $\mu$ is any probability measure on $\mathbb{R}^{n}$. Recently Talagrand's inequality and its counterpart, the logarithmic Sobolev inequality (LSI) have received a lot of attention and they have been extended from the Euclidean spaces to Riemannian manifolds. (Unlike Talagrand's inequality, the LSI gives an upper bound for the relative entropy.) It was shown by F. Otto and C. Villani [12] that in the Riemannian manifold setting the TCI follows from the LSI due to D. Bakry and M. Emery [1].

On the other hand, Ph. Biane and D. Voiculescu [3] proved the free analogue of Talagrand's TCI for compactly supported measures on the real line. They replaced the relative entropy with the relative free entropy and the Gaussian measure with the semicircular law. Based on the method of random matrix approximation, Biane [2] proved the free LSI for measures on the real line, and we made a slight generalization of Biane and Voiculescu's free TCI [8]. We also obtained the free TCI and LSI for measures on the unit circle using large deviation results for special unitary matrices and the differential geometry of $\mathrm{SU}(n)[8,9]$.

Recently M. Ledoux [10] used the random matrix method to obtain the free analogue of the Prékopa-Leindler inequality on the real line. From this together with the HamiltonJacobi approach, he also gave different proofs of the free LSI and TCI for measures on $\mathbb{R}$. The aim of the present note is to give a new proof of the free TCI for measures on the circle following Ledoux's idea. In this way we do not need the large deviation technique but we establish a kind of free analogue of Prékopa-Leindler inequality on the circle.

1. The Prékopa-Leindler inequality on a Riemannian manifold. Let $M$ be a complete, connected, $n$-dimensional Riemannian manifold with the volume measure $d x$ and the geodesic distance $d(x, y)$ for $x, y \in M$. For $0<\theta<1$ define

$$
Z_{\theta}(x, y):=\{z \in M: d(x, z)=\theta d(x, y), d(z, y)=(1-\theta) d(x, y)\}
$$

which is the locus of points playing the role of $(1-\theta) x+\theta y$. In this section we first present a result of Cordero-Erausquin, McCann and Schmuckenschläger, which is an extension of the Prékopa-Leindler inequality to the Riemannian manifold setting. Then we show that this results implies the TCI on a Riemannian manifold under some conditions (slightly stronger than the Bakry-Emery criterion).

TheOREM 1.1 ([5, Corollary 1.2]). Assume that $\operatorname{Ric}(M) \geq(n-1) k$ holds for some $k \in \mathbb{R}$ where $\operatorname{Ric}(M)$ is the Ricci curvature of $M$. Let $f, g, h: M \rightarrow[0, \infty)$ be Borel measurable functions and fix $0<\theta<1$. Assume that

$$
h(z) \geq\left(\frac{S_{k}(d)}{S_{k}((1-\theta) d)^{1-\theta} S_{k}(\theta d)^{\theta}}\right)^{n-1} f(x)^{1-\theta} g(y)^{\theta}
$$

holds for every $x, y \in M, z \in Z_{\theta}(x, y)$ and $d:=d(x, y)$, where

$$
S_{k}(d):= \begin{cases}\sin (\sqrt{k} d) / \sqrt{k} d & \text { if } k>0, \\ 1 & \text { if } k=0, \\ \sinh (\sqrt{-k} d) / \sqrt{-k} d & \text { if } k<0 .\end{cases}
$$


Then

$$
\int_{M} h(x) d x \geq\left(\int_{M} f(x) d x\right)^{\theta}\left(\int_{M} g(x) d x\right)^{1-\theta} .
$$

Here it is worth noting a known result: If $\operatorname{Ric}(M) \geq(n-1) k$ with $k>0$, then the diameter of $M$ is at most $\pi / \sqrt{k}$ (see $[4,1.26]$ ).

Write

$$
\Phi_{\theta}(d):=(n-1)\left(\log S_{k}(d)-(1-\theta) \log S_{k}((1-\theta) d)-\theta \log S_{k}(\theta d)\right) .
$$

Let $\mathcal{M}(M)$ denote the set of probability Borel measures on $M$. Let $\nu \in \mathcal{M}(M)$ be given by $d \nu:=\frac{1}{Z} e^{-Q(x)} d x$ with a Borel function $Q: M \rightarrow \mathbb{R}$ and a normalization constant $Z$. Write

$$
R_{\theta}(z ; x, y):=Q(z)-(1-\theta) Q(x)-\theta Q(y) .
$$

Then, the above theorem is rephrased as follows:

If $u, v, w: M \rightarrow \mathbb{R}$ are Borel functions and

$$
w(z) \geq(1-\theta) u(x)+\theta v(y)+R_{\theta}(z ; x, y)+\Phi_{\theta}(d)
$$

holds for every $x, y \in M, z \in Z_{\theta}(x, y)$ and $d:=d(x, y)$, then

$$
\log \int_{M} e^{w(x)} d \nu(x) \geq(1-\theta) \log \int_{M} e^{u(x)} d \nu(x)+\theta \log \int_{M} e^{v(x)} d \nu(x) .
$$

The following transportation cost inequality in the Riemannian setting was shown in [12] based on [1].

Theorem $1.2\left([1]\right.$ and [12]). Let $\nu \in \mathcal{M}(M)$ be given by $d \nu(x):=\frac{1}{Z} e^{-Q(x)} d x$ with a $C^{2}$ function $Q: M \rightarrow \mathbb{R}$. If the Bakry and Emery criterion

$$
\operatorname{Ric}(M)+\operatorname{Hess}(Q) \geq \rho I_{n}
$$

is satisfied with a constant $\rho>0$, then

$$
W(\mu, \nu) \leq \sqrt{\frac{1}{\rho} S(\mu, \nu)}, \quad \mu \in \mathcal{M}(M) .
$$

Now, we assume the following condition slightly stronger than (1.2):

$$
\operatorname{Ric}(M) \geq \alpha I_{n} \quad \text { and } \operatorname{Hess}(Q) \geq \beta I_{n}
$$

for some constants $\alpha \geq 0, \beta \in \mathbb{R}$ with $\alpha+\beta=\rho>0$. Here, we assume $\alpha=0$ in case of $n=1$. Our goal in this section is to prove that Theorem 1.1 implies Theorem 1.2 under the assumption (1.3).

We use the celebrated variational formula (or the Monge-Kantorovich duality) for the Wasserstein distance (see [16]):

$$
\begin{aligned}
& \rho W(\mu, \nu)^{2}=\sup \left\{\int_{M} f(x) d \mu(x)-\int_{M} g(x) d \nu(x):\right. \\
& \left.\quad f, g \in C_{b}(M), f(x) \leq g(y)+\frac{\rho}{2} d(x, y)^{2}, x, y \in M\right\}
\end{aligned}
$$

where $\rho>0$. The variational expression for the relative entropy is also useful:

$$
S(\mu, \nu)=\sup \left\{\int_{M} f(x) d \mu(x)-\log \int_{M} e^{f(x)} d \nu(x): f \in C_{b}(M)\right\} .
$$

Furthermore, we need the Taylor expansion of $\log S_{k}(d)$ : 
LEMma 1.3. For $k \geq 0$,

$$
\log S_{k}(d)=-\sum_{j=1}^{\infty} c_{j}\left(k d^{2}\right)^{j}
$$

with $c_{1}=\frac{1}{6}$ and $c_{j}>0$ for all $j \geq 1$.

Proof. Set $f(x):=\log \frac{\sin x}{x}$; then we have

$$
f^{\prime}(x)=\cot x-\frac{1}{x}=\sum_{m=1}^{\infty} \frac{2 x}{x^{2}-(m \pi)^{2}}
$$

because of the well-known expansion of $\cot x$. Since

$$
f(x)=-\sum_{m=1}^{\infty} \frac{1}{m \pi}\left(\frac{1}{1-\frac{x}{m \pi}}-\frac{1}{1+\frac{x}{m \pi}}\right)=-\sum_{m=1}^{\infty} \sum_{j=1}^{\infty} \frac{2}{(m \pi)^{2 j}} x^{2 j-1},
$$

we get

$$
f^{(2 j)}(0)=-\frac{2(2 j-1) !}{\pi^{2 j}} \sum_{m=1}^{\infty} \frac{1}{m^{2 j}}
$$

so that

$$
c_{j}=-\frac{f^{(2 j)}(0)}{(2 j) !}=\frac{1}{j \pi^{2 j}} \sum_{m=1}^{\infty} \frac{1}{m^{2 j}}>0 .
$$

Since $\operatorname{Ric}(M) \geq(n-1) k$ with $k=\frac{\alpha}{n-1}$ due to the assumption $\operatorname{Ric}(M) \geq \alpha I_{n}$ in $(1.3)$, we get by Lemma 1.3

$$
\begin{aligned}
\Phi_{\theta}(d) & =-(n-1) \sum_{j=1}^{\infty}\left(1-(1-\theta)^{2 j+1}-\theta^{2 j+1}\right) c_{j}\left(k d^{2}\right)^{j} \\
& \leq-(n-1)\left(1-\theta^{3}-(1-\theta)^{3}\right) \frac{\alpha}{6(n-1)} d^{2} \\
& =-\frac{\alpha \theta(1-\theta)}{2} d^{2} .
\end{aligned}
$$

For each $x, y \in M$ let $z(t)(0 \leq t \leq 1)$ be a geodesic curve joining $x, y$ with $d(x, z(t))=$ $t d(x, y)$. Since the assumption $\operatorname{Hess}(Q) \geq \beta I_{n}$ in (1.3) gives

we get

$$
\frac{d^{2}}{d t^{2}} Q(z(t)) \geq \beta d(x, y)^{2}, \quad 0 \leq t \leq 1,
$$

$$
\begin{aligned}
R_{\theta}(z(\theta) ; x, y) & =Q(z(\theta))-\theta Q(z(0))-(1-\theta) Q(z(1)) \\
& \leq-\frac{\beta \theta(1-\theta)}{2} d(x, y)^{2} .
\end{aligned}
$$

Hence, by (1.6) and (1.7) we have

$$
R_{\theta}(z ; x, y)+\Phi_{\theta}(d(x, y)) \leq-\frac{\rho \theta(1-\theta)}{2} d(x, y)^{2}
$$

for every $x, y \in M$ and $z \in Z_{\theta}(x, y)$.

Now, let $f, g \in C_{b}(M)$ be such that

$$
f(x) \leq g(y)+\frac{\rho}{2} d(x, y)^{2}, \quad x, y \in M .
$$


Set $u:=\theta f, v:=-(1-\theta) g$ and $w:=0$. Then

$$
\begin{aligned}
& (1-\theta) u(x)+\theta v(y)+R_{\theta}(z ; x, y)+\Phi_{\theta}(d(x, y)) \\
& \quad \leq \theta(1-\theta)\left\{f(x)-g(y)-\frac{\rho}{2} d(x, y)^{2}\right\} \leq 0=w(z)
\end{aligned}
$$

for every $x, y \in M$ and $z \in Z_{\theta}(x, y)$. Hence Theorem 1.1 (the rephrased version (1.1)) yields

$$
\log \int_{M} e^{\theta f(x)} d \nu(x)+\frac{\theta}{1-\theta} \log \int_{M} e^{-(1-\theta) g(x)} d \nu(x) \leq 0 .
$$

Letting $\theta \nearrow 1$ gives

$$
\log \int_{M} e^{f(x)} d \nu(x)-\int_{M} g(x) d \nu(x) \leq 0
$$

so that

$$
\int_{M} f(x) d \mu(x)-\int_{M} g(x) d \nu(x) \leq \int_{M} f(x) d \mu(x)-\log \int_{M} e^{f(x)} d \nu(x) \leq S(\mu, \nu)
$$

thanks to (1.5). Finally, we apply (1.4) to obtain

$$
\rho W(\mu, \nu)^{2} \leq S(\mu, \nu)
$$

2. Free TCI on the circle. Let $Q: \mathbb{T} \rightarrow \mathbb{R}$ be a continuous function. The weighted energy integral associated with $Q$ is defined by

$$
E_{Q}(\mu):=-\Sigma(\mu)+\int_{\mathbb{T}} Q(\zeta) d \mu(\zeta) \quad \text { for } \mu \in \mathcal{M}(\mathbb{T})
$$

which admits a unique minimizer $\nu_{Q} \in \mathcal{M}(\mathbb{T})$ (see [14]). Set $B(Q):=-E_{Q}\left(\nu_{Q}\right)$ and define the relative free entropy with respect to $Q$ by

$$
\widetilde{\Sigma}_{Q}(\mu):=-\Sigma(\mu)+\int_{\mathbb{T}} Q(\zeta) d \mu(\zeta)+B(Q) \text { for } \mu \in \mathcal{M}(\mathbb{T}) .
$$

It is known ([9, Theorem 2.1], also [7, Chap. 5]) that $\widetilde{\Sigma}_{Q}(\mu)$ is the rate function of the large deviation principle (in the scale $1 / N^{2}$ ) for the empirical eigenvalue distribution of the special unitary random matrix

$$
d \lambda_{N}^{\mathrm{SU}}(Q)(U):=\frac{1}{Z_{N}^{\mathrm{SU}}(Q)} \exp \left(-N \operatorname{Tr}_{N}(Q(U))\right) d U
$$

where $d U$ is the Haar probability measure on the special unitary group $\mathrm{SU}(N)$ of order $N, Q(U)$ for $U \in \mathrm{SU}(N)$ is defined via functional calculus and $\operatorname{Tr}_{N}$ is the usual trace on the $N \times N$ matrices.

The Wasserstein distance $W(\mu, \nu)$ between $\mu, \nu \in \mathcal{M}(\mathbb{T})$ is defined with respect to the angular distance (i.e., the geodesic distance). The following is the free TCI for measures on $\mathbb{T}$ proven in [8]. The aim of this section is to re-prove this by using the method of Ledoux [10].

TheOREM 2.1 ([8, Theorem 2.7]). Let $Q: \mathbb{T} \rightarrow \mathbb{R}$ be a continuous function. If there exists a constant $\rho>-\frac{1}{2}$ such that $Q\left(e^{\mathrm{i} t}\right)-\frac{\rho}{2} t^{2}$ is convex on $\mathbb{R}$, then

$$
W\left(\mu, \nu_{Q}\right) \leq \sqrt{\frac{2}{1+2 \rho} \widetilde{\Sigma}_{Q}(\mu)}, \quad \mu \in \mathcal{M}(\mathbb{T}) .
$$


We introduce the relative free pressure with respect to $Q$ by

$$
j_{Q}(f):=\sup \left\{\int_{\mathbb{T}} f d \mu-\widetilde{\Sigma}_{Q}(\mu): \mu \in \mathcal{M}(\mathbb{T})\right\} \quad \text { for } f \in C_{\mathbb{R}}(\mathbb{T}) .
$$

It is known ([10] and [6]) that

$$
\begin{aligned}
j_{Q}(f) & =E_{Q}\left(\nu_{A}\right)-E_{Q-f}\left(\nu_{Q-f}\right) \\
& =\lim _{N \rightarrow \infty} \frac{1}{N^{2}} \log \int_{\mathrm{SU}(N)} \exp \left(N \operatorname{Tr}_{N}(f(U))\right) d \lambda_{N}^{\mathrm{SU}}(Q)(U) .
\end{aligned}
$$

For $N \in \mathbb{N}$ and $U, V, W \in \mathrm{SU}(N)$ write

$$
R_{\theta, N}(W ; U, V):=\operatorname{Tr}_{N}(Q(W))-(1-\theta) \operatorname{Tr}_{N}(Q(U))-\theta \operatorname{Tr}_{N}(Q(V)) .
$$

The next lemma is a sort of free analogue of Prékopa-Leindler-Ledoux inequality on the circle.

Lemma 2.2. Let $f, g, h: \mathbb{T} \rightarrow \mathbb{R}$ be Borel functions and fix $0<\theta<1$. Assume that

$$
\begin{aligned}
\operatorname{Tr}_{N}(h(W)) \geq(1-\theta) \operatorname{Tr}_{N}(f(U))+\theta \operatorname{Tr}_{N}(g(V)) & \\
& +R_{\theta, N}(W ; U, V)-\frac{\theta(1-\theta)}{4} d(U, V)^{2}
\end{aligned}
$$

holds for every $N \in \mathbb{N}, U, V \in \mathrm{SU}(N)$ and $W \in Z_{\theta}(U, V)$. Then

$$
j_{Q}(h) \geq(1-\theta) j_{Q}(f)+\theta j_{Q}(g) .
$$

Proof. Since $\operatorname{dim}(\mathrm{SU}(N))=N^{2}-1$ and $\operatorname{Ric}(\mathrm{SU}(N))=\frac{N}{2}, \Phi_{\theta}$ defined for $M=\mathrm{SU}(N)$ satisfies

$$
\Phi_{\theta}(d) \leq-\frac{N \theta(1-\theta)}{4} d^{2}
$$

thanks to (1.6). Hence, for each $N \in \mathbb{N}$, the assumption of the lemma gives

$$
\begin{aligned}
N \operatorname{Tr}_{N}(h(W)) \geq(1-\theta) & N \operatorname{Tr}_{N}(f(U))+\theta N \operatorname{Tr}_{N}(g(V)) \\
& +N R_{\theta, N}(W ; U, V)+\Phi_{\theta}(d(U, V))
\end{aligned}
$$

for every $U, V \in \mathrm{SU}(N)$ and $W \in Z_{\theta}(U, V)$. Theorem 1.1 (the rephrased version (1.1)) can be applied to $\nu:=\lambda_{N}^{\mathrm{SU}}(Q), u:=N \operatorname{Tr}_{N}(f(\cdot)), v:=N \operatorname{Tr}_{N}(g(\cdot))$ and $w:=N \operatorname{Tr}_{N}(h(\cdot))$; hence we have

$$
\begin{aligned}
& \log \int_{\mathrm{SU}(N)} \exp \left(N \operatorname{Tr}_{N}(h(U))\right) d \lambda_{N}^{\mathrm{SU}}(Q)(U) \\
& \geq(1-\theta) \log \int_{\mathrm{SU}(N)} \exp \left(N \operatorname{Tr}_{N}(f(U))\right) d \lambda_{N}^{\mathrm{SU}}(Q)(U) \\
& \quad+\theta \log \int_{\mathrm{SU}(N)} \exp \left(N \operatorname{Tr}_{N}(g(U))\right) d \lambda_{N}^{\mathrm{SU}}(Q)(U)
\end{aligned}
$$

implying the inequality (2.2) thanks to (2.1).

The assumption of the lemma is apparently too much; so the above must not be the optimal form of the free Brunn-Minkowski inequality on $\mathbb{T}$. Nevertheless, it is enough to prove Theorem 2.1. 
For each $N \in \mathbb{N}$ and $U \in \mathrm{SU}(N)$ set $\Psi(U):=\operatorname{Tr}_{N}(Q(U))$. Using a certain regularization technique as in [8], we may assume that $Q$ is a harmonic function in a neighborhood of the unit disk. Then, it was shown in [8, Lemma 1.3 (ii)] that the convexity assumption of $Q$ implies $\operatorname{Hess}(\Psi) \geq \rho I_{N^{2}-1}$. This gives as in (1.7)

$$
R_{\theta, N}(W ; U, V) \leq-\frac{\rho \theta(1-\theta)}{2} d(U, V)^{2}
$$

for every $U, V \in \mathrm{SU}(N)$ and $W \in Z_{\theta}(U, V)$. Now, let $f, g \in C(\mathbb{T})$ be such that

$$
f(\zeta) \leq g(\eta)+\frac{1+2 \rho}{4} d(\zeta, \eta)^{2}, \quad \zeta, \eta \in \mathbb{T} .
$$

Define the optimal matching distance on $\mathbb{T}^{N}$ by

$$
\delta(\zeta, \eta):=\min _{\sigma \in S_{N}} \sqrt{\sum_{i=1}^{N} d\left(\zeta_{i}, \eta_{\sigma(i)}\right)^{2}}
$$

for $\zeta=\left(\zeta_{1}, \ldots, \zeta_{N}\right), \eta=\left(\eta_{1}, \ldots, \eta_{N}\right) \in \mathbb{T}^{N}$. For $U \in \mathrm{SU}(N)$ let $\lambda(U):=\left(\lambda_{1}(U), \ldots, \lambda_{N}(U)\right)$ denote the element of $\mathbb{T}^{N}$ consisting of the eigenvalues of $U$ with multiplicities and in counter-clockwise order. It immediately follows from (2.4) that

$$
\operatorname{Tr}_{N}(f(U)) \leq \operatorname{Tr}_{N}(g(V))+\frac{1+2 \rho}{4} \delta(\lambda(U), \lambda(V))^{2}, \quad U, V \in \mathrm{SU}(N) .
$$

Since $\delta(\lambda(U), \lambda(V)) \leq d(U, V)$ as shown in $[8,(2.11)])$, this gives

$$
\operatorname{Tr}_{N}(f(U)) \leq \operatorname{Tr}_{N}(g(V))+\frac{1+2 \rho}{4} d(U, V)^{2}, \quad U, V \in \mathrm{SU}(N) .
$$

Set $\tilde{f}:=\theta f, \tilde{g}:=-(1-\theta) g$ and $\tilde{h}:=0$. Then, for $U, V \in \operatorname{SU}(N)$ and $W \in Z_{\theta}(U, V)$, by (2.3) and (2.5) we get

$$
\begin{aligned}
& (1-\theta) \operatorname{Tr}_{N}(\tilde{f}(U))+\theta \operatorname{Tr}_{N}(\tilde{g}(V))+R_{\theta, N}(W ; U, V)-\frac{\theta(1-\theta)}{4} d(U, V)^{2} \\
& \quad \leq \theta(1-\theta)\left(\operatorname{Tr}_{N}(f(U))-\operatorname{Tr}_{N}(g(V))-\frac{1+2 \rho}{4} d(U, V)^{2}\right) \\
& \quad \leq 0=\operatorname{Tr}_{N}(\tilde{h}(W)) .
\end{aligned}
$$

Hence, the assumption of Lemma 2.2 is satisfied so that we have

$$
(1-\theta) j_{Q}(\theta f)+\theta j_{Q}(-(1-\theta) g) \leq j_{Q}(0)=0 .
$$

For every $\mu \in \mathcal{M}(\mathbb{T})$, by definition of $j_{Q}$, this implies

$$
(1-\theta)\left(\int_{\mathbb{T}} \theta f d \mu-\widetilde{\Sigma}_{Q}(\mu)\right)+\theta\left(\int_{\mathbb{T}}(-(1-\theta) g) d \nu_{Q}-\widetilde{\Sigma}_{Q}\left(\nu_{Q}\right)\right) \leq 0
$$

so that, thanks to $\widetilde{\Sigma}_{Q}\left(\nu_{Q}\right)=0$,

$$
\theta\left(\int_{\mathbb{T}} f d \mu-\int_{\mathbb{T}} f d \nu_{Q}\right) \leq \widetilde{\Sigma}_{Q}(\mu)
$$

Letting $\theta \nearrow 1$ gives

$$
\int_{\mathbb{T}} f d \mu-\int_{\mathbb{T}} f d \nu_{Q} \leq \widetilde{\Sigma}_{Q}(\mu) .
$$


Using (1.4) we obtain

$$
\frac{1+2 \rho}{2} W\left(\mu, \nu_{Q}\right)^{2} \leq \widetilde{\Sigma}_{Q}(\mu)
$$

It turns out that the bound $2 /(1+2 \rho)$ of our free TCI on $\mathbb{T}$ cannot be improved even if we use the Riemannian Prékopa-Leindler inequality from [5]. This suggests the best possibility of the bound.

Acknowledgments. We are grateful to Professor M. Ledoux for sending us his preprint version of [10], to Professor Y. Ueda for suggesting us the proof of free TCI by using [5], and to Dr. A. Andai for the proof of Lemma 1.3.

\section{References}

[1] D. Bakry and M. Emery, Diffusion hypercontractives, in: Séminaire de Probabilités XIX, Lecture Notes in Math. 1123, Springer-Verlag, 1985, 177-206.

[2] Ph. Biane, Logarithmic Sobolev inequalities, matrix models and free entropy, Acta Math. Sinica 19 (2003), 1-11.

[3] Ph. Biane and D. Voiculescu, A free probabilistic analogue of the Wasserstein metric on the trace-state space, Geom. Funct. Anal. 11 (2001), 1125-1138.

[4] J. Cheeger and D. G. Ebin, Comparison Theorems in Riemannian Geometry, NorthHolland, Amsterdam-Oxford; Elsevier, New York, 1975.

[5] D. Cordero-Erausquin, R. J. McCann and M. Schmuckenschläger, A Riemannian interpolation inequality à la Borell, Brascamp and Lieb, Invent. Math. 146 (2001), 219-257.

[6] F. Hiai, M. Mizuo and D. Petz, Free relative entropy for measures and a corresponding perturbation theory, J. Math. Soc. Japan 54 (2002), 679-718.

[7] F. Hiai and D. Petz, The Semicircle Law, Free Random Variables and Entropy, Mathematical Surveys and Monographs, 77, Amer. Math. Soc., Providence, 2000.

[8] F. Hiai, D. Petz and Y. Ueda, Free transportation cost inequalities via random matrix approximation, Probab. Theory Related Fields 130 (2004), 199-221.

[9] F. Hiai, D. Petz and Y. Ueda, A free logarithmic Sobolev inequality on the circle, Canad. Math. Bull., to appear.

[10] M. Ledoux, A (one-dimensional) free Brunn-Minkowski inequality, C. R. Acad. Sci. Paris Sér. I Math. 340 (2005), 301-304.

[11] L. Leindler, On a certain converse of Hölder inequality, in: Linear Operators and Approximation, Birkhäuser, Basel, 1972, 182-184.

[12] F. Otto and C. Villani, Generalization of an inequality by Talagrand and links with the logarithmic Sobolev inequality, J. Funct. Anal. 173 (2000), 361-400.

[13] A. Prékopa, On logarithmic concave measures and functions, Acta Sci. Math. (Szeged) 34 (1973), 335-343.

[14] E. B. Saff and V. Totik, Logarithmic Potentials with External Fields, Springer-Verlag, Berlin-Heidelberg-New York, 1997.

[15] M. Talagrand, Transportation cost for Gaussian and other product measures, Geom. Funct. Anal. 6 (1996), 587-600.

[16] C. Villani, Topics in Optimal Transportation, Grad. Studies in Math. 58, Amer. Math. Soc., Providence, 2003. 